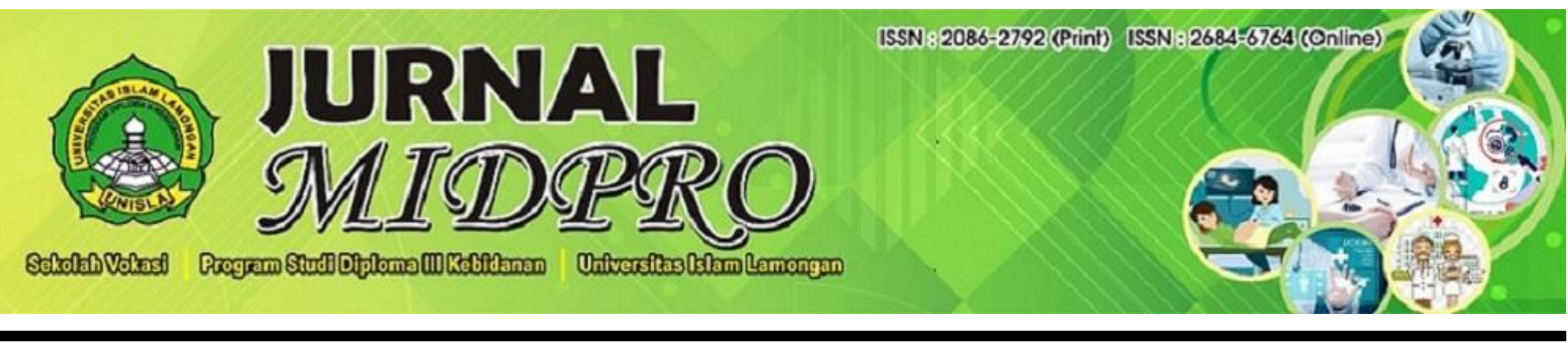

\title{
Serum Caspase-3 And Tissue Of Cervical Intraepithelial Neoplasia (CIN) In Wistar Rats With Diethylstilbestrol Induction
}

\author{
KSri Rahayu $^{1}$, Iting ${ }^{2}$ \\ ${ }^{1}$ Midwifery Program, Health Science, University Singaperbangsa Karawang \\ ${ }^{2}$ Nursing Program,STIKes Nurul Hasanah Kutacane Aceh Tenggara \\ Email Korespondensi $\left({ }^{\mathrm{K}}\right)$ : sri.rahayu@fikes.unsika.ac.id
}

\begin{abstract}
Diethylstilbestrol (DES) is a synthetic estrogen hormone that is useful as hormone replacement therapy, but longterm use of DES in cervical epithelium triggers cervical intraepithelial neoplasia (CIN). CIN can develop into cervical cancer. Cervical cancer is associated with the potential for invasion and metastasis, with the gold standard is histopathological examination. Accurate diagnosis and prediction of malignancy are important issues in clinical management, including the identification of biomarkers. Caspase or Cytosolic Aspartate-Specific Cysteine Proteases (caspase) are a group of proteases that facilitate apoptosis. Caspase- 3 is a key mediator of the apoptotic process that acts as an anti-cancer. Exposure to DES in rats can trigger cervical intraepithelial neoplasia. In stage CIN I, it does not need therapy because $80 \%$ of cell dysplasia can return to normal. In CIN II \& III stages can develop into cancer cells (true cervical cancers precursors). CIN is called pre-cancerous cells, and it can develop into cancerous if left untreated. However, not all women who experience CIN will suffer cancer. The study used female Wistar rats strains for 3-5 days in age, administration a DES dose $1500 \mu \mathrm{gram} / \mathrm{KgBB}$ for 35 days. Then examined caspase- 3 contained in serum and tissue through the ELISA method. The independent t-test result showed that there was no significant difference between caspase- 3 in serum and tissue with $\mathrm{p}>0.05$.
\end{abstract}

Keywords: CIN; Caspase-3; DES; Histologi; Serum

\section{Article history :}

Received: 17 Juni 2020

Received in revised form: 15 September 2020

Accepted: 10 October 2020

Available online: 1 December 2020

licensed by Creative Commons Attribution-ShareAlike 4.0 International License. 


\section{INTRODUCTION}

The annual number of new cervical cancer cases is expected to increase 1-5 times by $2030 .{ }^{[1]}$ 98\% of cervical cancers are related to the Human Papilloma Virus (HPV). HPV infection does not all develop into cervical cancer. it indicates that endogenous and exogenous factors affect the process of cervical cancer. $\cdot 1,2$

Various risk factors of cervical cancer include socio-demographic factors (age, socioeconomic status) and sexual activity factors (early age at first sexual intercourse and multiple sexual partners). Besides, chlamydia infection, immune suppression, parity, lack of maintaining genital hygiene, smoking, history of venereal disease, family history of cervical cancer, chronic cervical trauma, oral contraceptives, and diethylstilbestrol (DES). ${ }^{2,3}$

DES is a synthetic estrogen hormone that is useful as a hormone replacement therapy, but DES can also trigger abnormal cell growth that will develop into cervical cancer. ${ }^{4,5}$

DES or diethylstilbestrol is a synthesis of estrogen or xenoestrogen with the chemical formula C18H20O2. Pregnant women use DES to prevent miscarriages and preterm labor. In 1946-1971, between 2 and 4 million individuals were exposed to DES during pregnancy, and it causes a disorder known as DES daughters. In DES daughters, reproductive tract abnormalities were found, namely vaginal and cervical adenosis, followed by columnar epithelium development in the cervix and vagina. DES daughters are at risk for developing cervicovaginal clear cell-adenomas. Cervical vaginal adenosis as a precursor to adenocarcinoma. ${ }^{2,7}$

Cervical cancer is associated with the potential for invasion and metastasis, and the gold standard is histopathology. ${ }^{3,6}$. Cervical epithelial cell dysplasia was found with the standard cervical intraepithelial neoplasia (CIN) stage on histopathological examination. CIN is known as pre-cancerous cells, and it can develop into cancerous if left untreated for some time. However, not all women who have CIN will have cancer.

There are no specific symptoms of CIN. CIN examination reduces the risk of cervical cancer. ${ }^{6}$. CIN screening is an effective method to identify the presence of cancer to reduce mortality. However, there is a risk of recurrent $\mathrm{CIN}$ after treatment. Age, menopause, post-surgery is some of the recurrent CIN factors. ${ }^{7}$

CIN examination can reduce both the incidence and mortality of cancer. CIN can be detected and treated immediately. ${ }^{9}$ Pre-cancer treatment is more effective, efficient, and low cost before it develops into cancer cells. In stage CIN, It does not need therapy because $80 \%$ of cell dysplasia can normalize. ${ }^{6}$ CIN II \& III required therapy such as; laser, cryotherapy, LEEP (loop electrosurgical excision procedure), and laser conization. CIN II and III can develop into cancer cells (true cervical cancer precursors) within 12 months. ${ }^{6}$ 
Nearly 330,000 new cases of CIN were found in Europe, 50\% were CIN2 and CIN3. The results of the study, approximately 1.5 per 1000 women in developed countries are diagnosed as having CIN2/3 annually, and the incidence is highest among women aged between 25 and 29 years ${ }^{8}$

CIN screening is an effective method to identify the presence of cancer to reduce mortality. Accurate diagnosis and malignancy prediction are important clinical management issues, including identifying biomarkers for CIN and malignancy diagnosis. The biomarker in this study is caspase. Caspase is an endoprotease with an active site Cys (C) and cleaves at the C-terminal side of Asp residue. Therefore, it is known as caspases (Cys containing-Asp specific protease). Several members of the caspase family involved in apoptosis can be divided into 2 groups. The first group consists of caspase 8, caspase 9 , and caspase 10, which contain a long prodomain at the N-terminus and function as initiators of the cell death process. The second group consists of caspases 3, 6, 7, which contain short pro domains and function as effectors, cleaving various dead substrates, which in turn cause morphological and biochemical changes seen in apoptotic cells. Caspase-3 (CPP32, apopain, YAMA) is a key mediator of mammalian cells' apoptotic process.

Caspase is in an inactive state until one of the caspases is activated by a signal, then a series of reactions (cascade) of caspase activation occurs through a proteolytic process. ${ }^{9}$. Based on the research results, DES exposure resulted in the disruption of abnormal cell growth. Determining the objective biomarkers of malignancy is important. There for, an in vivo study examined serum caspase-3 and tissue on CIN by induction of diethylstilbestrol (DES) in female neonatal rats at the age of 3-5 days

\section{METHOD}

This research was experimental research conducted in vivo. It used a strain of female Wistar rats at 3-5 days of age to avoid endogenous estrogens' effects. The DES dose given was $1500 \mu \mathrm{gram} / \mathrm{kg}$ BW to trigger CIN. ${ }^{10}$

DES suspended in a corn oil solution was administered subcutaneously. 5 female Wistar rats at 3-5 days of age were given DES for 45 days at a dose of $1500 \mu$ gram / KgBB. After being exposed to DES, the rats were then taken blood serum to be measured caspase- 3 by ELISA. After that, the rats were turned off and examined for caspase-3 cervical epithelial tissue. Then, it observed epithelial cell dysplasia and grouped the $\mathrm{CIN}$ of those five rats.

\section{RESULTS}

The results of the study after DES administration, caspase-3 levels observation, and histological examination in cervical tissue can be seen in the following table: 
Table. 1 Frequency distribution of CIN staging after DES induction

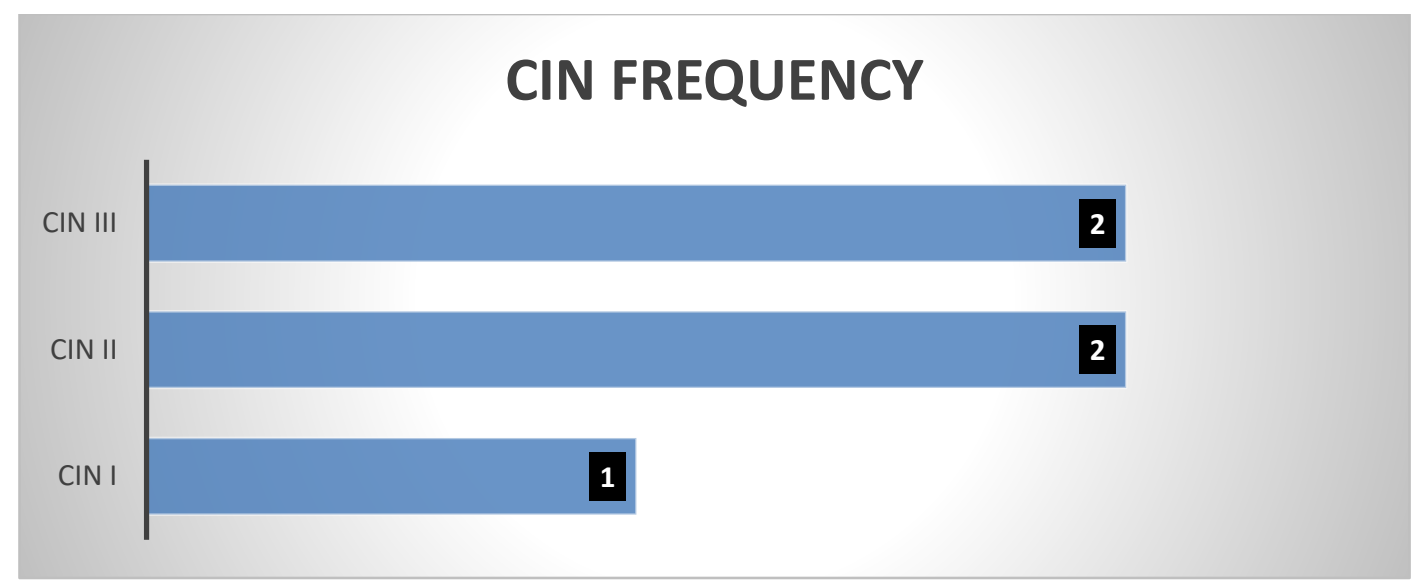

Table 1 shows that the Cervical Intraepithelial Neoplasm (CIN) stage after DES exposure is $100 \%$ experiencing CIN.

Table. 2 Mean of caspase- 3 in serum and tissue after 45 days of DES exposure

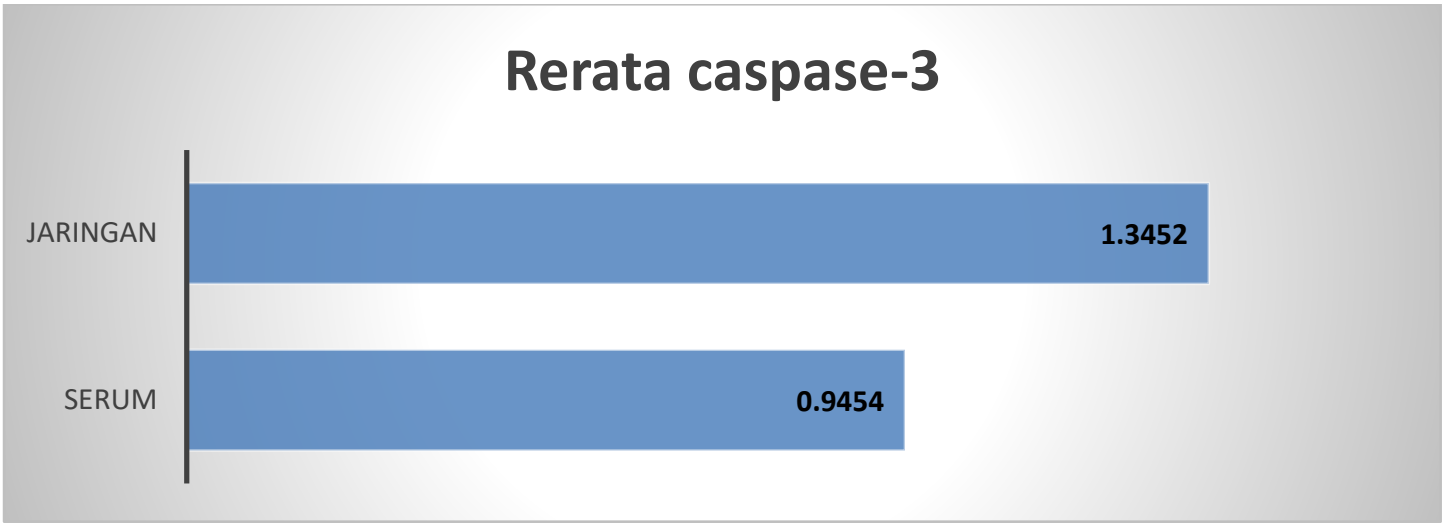

Table 2 illustrates the mean of caspase- 3 in serum and tissue. The mean of caspase- 3 was higher in tissue than in serum.

The independent $\mathrm{t}$-test result is $\mathrm{p}>0.05$. There was no significant difference between caspase- 3 in serum and caspase- 3 in tissue.

\section{DISCUSSION}

This study gave DES $100 \%$ caused CIN, even though the samples were neonatal rats. However, despite exposure to the same time and dose, the observed CIN stages were not at the same stage. DES has different effects depending on the strain, age, time, and DES dose.

Administration of DES at neonatal age minimizes endogenous estrogens' effects. Abnormal cell formation in the vaginal epithelium due to DES can increase protooncogenes: $c-J u n, c-F o s, c-M y c$, and lead to cancer. ${ }^{11}$. DES causes cervical cell dysplasia ${ }^{5}$. Estrogen causes cancer in non-physiological doses. Estrogens increase oxidation by damaging the DNA in estrogen-responsive tissues. ${ }^{12}$ 
DES exposure causes genotoxicity and carcinogenesis. Neonatal DES exposure can cause demethylate or hypomethylated, which causes DNA to be unstable and easily damaged. On chromosomes that are being rearranged, DES raises aneugenic potential and causes DNA binding protein to break and disrupt the satellite centromere region. Apart from that, DES also inhibits intrachain crosslinking in tubulin.

Estrogen is a hormone that has genotoxic potential. Its action is through the estrogen metabolite, quinone. Induced oxidation and aldehydes produce mutagenic effects at the apurinic site in DNA, leading to lipid peroxidation. DES also causes damage to mitochondria, particularly cytochrome c oxidase. DES causes cancer through a combination of epigenetics and genotoxins. Experts confirm the presence of estrogen receptor agonists that stimulate transcription and proliferation in target cells. There is a strong bond between the DNA nucleotide and the DES metabolite. DES increases DNA adduct, and it is called I-compounds, the generation of free radicals up to the mutation of p53 by DES. DES effect due to DNA adduct in women 4-6 times higher than men ${ }^{12}$. DES works by disrupting the neuroendocrine through the release of the hormone gonadotropin release hormone $(\mathrm{GnRH})$. DES will decrease kisspeptin mRNA levels or decrease GnRH stimulation in neurons stimulated by kisspeptin. Kisspeptin is a GnRH regulator. Kisspeptin regulates ovulation, the estrous cycle, sex differentiation and affects puberty ${ }^{10}$

DES is associated with protein 63 (TRP63), which is highly expressed in the squamous cervical epithelium and vagina. TRP63 affects squamosal differentiation in the Müllerian duct (MDE). The absence of TRP63 at birth causes defects in the development of the skin and reproductive system. DES exposure in experimental animals, expression of TRP63 in MDE was inhibited. In the critical phase, DES induces adenosis, causing failure of squamous differentiation in the MDE during organogenesis of the lower female reproductive tract. DES down-regulated Wnt7a in neonatal uteri, including differentiation of myometrium, lack of uterine glands, and squamous metaplasia. DES elicits its teratogenic effects via down-regulation of Wnt7a. Mutations in Wnt7a altered uterine expression of other developmental regulators, including Hoxa10 and Hoxa 11, which are also down-regulated in the neonatally DES uterus. his gene plays an important role in the development of Estrogen Receptor Alpha $(\operatorname{ER} \alpha){ }^{13}$

The independent $\mathrm{t}$-test result showed no significant difference between caspase- 3 in serum and caspase- 3 in tissue. It happened maybe because the sample was too small, then the CIN stages were different, so that caspase-3, which acted as apoptosis was also different. DES has a different effect on each rat. Thus it is not easy to find homogeneous respondents. Caspase- 3 is a biomarker that can be used in a clinical setting to assess for malignancy. Caspase- 3 is a key mediator of the apoptotic process in mammalian cells. The caspase is in an inactive state until one of the caspases is activated by a signal, then a cascade occurs, the next caspase activation is through a proteolytic process. ${ }^{[11]} \mathrm{Also}$, the number of respondents is too small, so the future study should use a larger sample to see caspase- 3 in serum and tissue, including serum and the tissue in normal standards. 
Caspase-3 is widely expressed in tissues. Its presence is normal in various types of tumor tissue. Normal cells contain a small amount of caspase-3. It is in the inactive form of the zymogen. The percentage of caspase-3 is decreased in cervical cancer compared to normal cervix and CIN. Decreased expression of caspase-3 due to apoptotic inhibition of cervical oncogenesis and development is used as a prognosis for cervical cancer malignancy. The expression of caspase- 3 was different in cervical cancer metastases/invasion. In non-lymph node metastases, the expression of caspase-3 was higher than in lymph node metastases. The expression of caspase-3 in cervical cancer can be used as a biomarker for diagnosing and prognosis of cervical cancer.

The expression of caspase- 3 has decreased in cervical cancer, especially CIN II and III. This reduction is important in assessing the progression of tumor formation and the development of cervical cancer. Thus, this condition can be used as predictor biomarkers to predict the development of cervical cancer $^{14}$

\section{CONCLUSION}

Various DES pathways cause disturbances in the reproductive system. It is necessary to monitor DES in various hormone therapies and estrogen in various other therapies. Further research is needed to examine caspase-3 on CIN with the same stage (same level), for example, all rats with stage CIN II or stage III. then a larger sample so that the respondents obtained is more homogeneous. In the future, this biomarker may serve as a reference for determining CIN standards without having a histopathological examination, which requires a longer time and a high human error rate. A more objective, practical, and modern standard is needed to diagnose CIN stages.

\section{REFERENCES}

1. Tsikouras P, Zervoudis S, Manav B, et al. Cervical cancer : screening, diagnosis and staging. JBUON. 2016;21(2):320-325.

2. Gracy G, Sadhna K, Jacqueline J, Deepika K. Highlights Of P53 Mutation And It 's Role In Cervical Cancer Metastasis. Int J Biol Med Res. 2014;3(1):3772-3779. doi:3772-3779

3. Ci A, Ne M, Po E. Gynecology \& Obstetrics Cervical Cancer : A Health Limiting Condition. Gynecol Obstet. 2016;6(5). doi:10.4172/2161-0932.1000378

4. Laronda MM, Unno K, Butler LM, Kurita T. The development of cervical and vaginal adenosis as a result of diethylstilbestrol exposure in utero. Differentiation. 2012;84(3):252-260. doi:10.1016/j.diff.2012.05.004

5. Yamashita S, Shimizu N. Effects of neonatal diethylstilbestrol exposure on c-fos and c-jun protooncogene expression in the mouse uterus. Histol Histopathol Cell Mol Biol Eff. Published online 2001:131-140.

6. Mitildzans A, Arechvo A, Rezeberga D, Isajevs S. Expression of p63, p53 and Ki-67 in patients with cervical intraepithelial neoplasia. Turk Patoloji Derg. 2017;33(1):9-16. doi:10.5146/tjpath.2016.01373

7. Sungwook Chun1 el all. The Neutrophil-Lymphocyte Ratio Predicts Recurrence of Cervical 
Intraepithelial Neoplasia. J Cancer. 2017;8(12). doi:10.7150/jca.19173

8. Suneela Vegunta, MD; Julia A. Files, MD; and Megan N. Wasson D. Screening Women at High Risk for Cervical. Mayo Clin Proc. 2017;92(8):1272-1277. doi:10.1016/j.mayocp.2017.06.007

9. $\quad \mathrm{Hu}$ Q, Peng J, Liu W, et al. Elevated cleaved caspase-3 is associated with shortened overall survival in several cancer types. Int J Clin Exp Pathol. 2014;7(8):5057-5070.

10. Yoshida M, Takahashi M, Inoue K, Hayashi S, Maekawa A, Nishikawa A. Delayed adverse effects of neonatal exposure to diethylstilbestrol and their dose dependency in female rats. Toxicol Pathol. 2011;39(5):823-834. doi:10.1177/0192623311413785

11. Iguchi T, Fukazawa Y, Bern HA. Effects of sex hormones on oncogene expression in the vagina and on development of sexual dimorphism of the pelvis and anococcygeus muscle in the mouse. Environ Health Perspect. 1995;103(SUPPL. 7):79-82. doi:10.2307/3432513

12. Fučić A, Stojkovic R, Katić J, et al. Animal model for age- and sex-related genotoxicity of diethylstilbestrol. Brazilian J Med Biol Res. 2009;42(11):1090-1096. doi:10.1590/S0100879X2009001100015

13. Kurita T, Mills AA, Cunha GR. Roles of p63 in the diethylstilbestrol-induced cervicovaginal adenosis. Dev Dis. 2004;131:1639-1649. doi:10.1242/dev.01038

14. Xia L, Xue XZ. Immunohistochemical study of NF-kB p65, c-IAP2 and caspase-3 expression in cervical cancer. Oncol Lett. 2012;3(4):839-844. doi:10.3892/ol.2012.564 